\title{
REMARQUES CONCERNANT L'HISTOIRE DES SUBORDONNÉES D'EXCEPTION EN LATIN ET DANS LES LANGUES ROMANES
}

La catégorie des subordonnées d'exception n'est pas présentée dans la plupart des grammaires de la langue latine.

Les traités de grammaire latine qui incluent quelques données sur ce sujet n'offrent aucune définition de la subordonnée d'exception. Ce qui est encore plus grave c'est que la subordonnée d'exception est d'habitude confondue avec la conditionnelle, si ce n'est, parfois, avec la proposition complétive, ou la comparative.

Les grammaires des langues romanes et des langues germaniques traitent de la subordonnée d'exception de manière incomplète et, le plus souvent, erronée. Le complément d'exception est lui aussi présenté de manière insatisfaisante dans les grammaires du latin et des langues néo-latines.

Nous allons commencer par dire que la subordonnée d'exception est beaucoup employée en latin à toutes les époques (à partir de l'époque préclassique), dans le registre populaire et dans le registre cultivé. La subordonnée d'exception apparaît dans des expressions proverbiales (voir Plaute, Trin., 439), dans des textes de lois, de senatusconsulta, dans le langage des juristes (comme par exemple celui de Q. Mucius Scaevola), dans les traités de médecine, d'agriculture, partout où une grande précision sémantique était nécessaire. La subordonnée d'exception est également fréquente dans l'éloquence païenne et chrétienne, dans la dramaturgie d'expression latine.

Les types les plus anciens de la subordonnée d'exception sont, selon toute vraisemblance, ceux introduits par nisi et quam, en dépendance d'une régente renfermant une négation (qu'il s'agisse de non, haud, nec, et ne-quidem attachés au verbe, ou qu'il s'agisse de pronoms, d'adjectifs, ou d'adverbes tels que: nemo, nihil, nequis, numquam, nusquam etc.).

Nisi-conjonction ancienne, spécifique de la subordonnée conditionnelle, disposait d'un sens restrictif nettement exprimé, autant à cause de l'adverbe-conjonction $s i$, que par la présence de la négation $n i<n e$. Quam était l'un des plus importants subordonnants de la proposition comparative. Son sens restrictif était formé depuis longtemps, dans le cadre de la comparaison d'inégalité. Ajoutons que la régente négative a beaucoup aidé à la naissance des subordonnées d'exception.

Nisi indique, au commencement de l'histoire des subordonnées d'exception, l'exclusion ou l'isolation d'une condition (cfr. Grec ancien: $\varepsilon \dot{\imath} \mu \eta$ ). Plus tard, nisi exprime l'exclusion d'un fait par rapport à celui de la proposition régente, tout en gardant sa 
capacité d'indiquer l'exclusion d'une condition. Quam (seule, ou en locution conjonctive avec $u t$ ) indique l'exclusion d'un fait; quam si, assez rare à l'époque préclassique, était employée pour l'exclusion d'une condition.

Voir par exemple:

- «... nil dixit, tu ut sequerere sese? //

nil (s.-en.: dixit), nisi abiens mi innuit...», Térence, Eun., 735-6.

- «nil est quod malim quam (s.-en.-: malo) illam totam familiam dari mi obuiam.», Térence, Ad., 311.

Cfr. à l'époque classique:

«Nos nihil de eo percontationibus reperiebamus, nisi certis ex aqua mensuris breuiores esse quam in continenti noctes uidebamus.», César, G. 5, 13, 4.

Pour les subordonnées exceptives introduites par nisi, à l'époque préclassique, voir aussi Plaute, Trin., 483. Pour ce qui est de l'emploi exceptif de nisi à l'époque classique, voir Cicéron, Fam., 13, 1, 4; R. Am., 108; César, C., 3, 44, 1 etc. Voir la discussion infra sur l'emploi de nisi à l'époque classique.

L'emploi de quam est conditionné par l'existence, dans la proposition régente, d'un mot présupposant une comparaison - d'habitude d'inégalité, de différence ou de préférence (des mots tels que: alius, aliter, contra, minor, peior; des verbes tels que malo, praestat, des expressions comme «melius est»; voir dans la citation de Térence, Ad., 311, le verbe malo). Des adjectifs et des adverbes indiquant l'égalité, la ressemblance ou la conformité peuvent aussi entrer en corrélation avec quam comparatif-exceptif, à condition que le verbe recteur soit négatif (cfr. Cicéron, Lael., 15). Les subordonnées comparatives-exceptives introduites par quam régies par des verbes négatifs, ne comportent pas de négation (nous parlons seulement des propositions introduites par quam et certaines locutions: quam ut, quam quod, quam si, quam qui; la négation est possible dans les subordonnées introduites par les locutions basées sur l'adverbe praeter quam, ou sur extra quam).

En ce qui concerne nisi, cette conjonction implique de manière évidente la négation. Elle doit être traduite par: «si ce n'est toutefois que + verbe affirmatif», ou par: «excepté $s i$ + verbe affirmatif», ou bien par: «excepté que, sauf que + verbes affirmatifs».

Nous tenons à préciser que le subordonnant restrictif quam apparaît souvent, à l'époque préclassique et aux époques suivantes, dans des locutions à sens essentiellement exceptif: praeter quam quod, praeter quam qui, extra quam si et d'autres encore. Exempli gratia: «ea (fana) exaugurauit, praeter quam quod Termino fanum fuit.», Caton, Hist., 24; ou:

- «at id, praeterquam quod fieri non potuit, ne fingi quidem potest.», Cicéron, Diu., 2, 28.

Voir aussi la discussion infra sur les locutions de nisi et de quam. 
La négation se trouve parfois contenue dans des adjectifs, ou des adverbes, à fonction d'attribut dans la régente (quelquefois il s'agit de litotes). Par exemple:

- « ... quid sit negoti, falsus incertusque sum, //

Nisi quia timeo tamen. ...», Plaute, Truc., 785-6.

La négation se trouve parfois rendue par des syntagmes nominaux - à fonction d'attribut: «extremae dementiae est ....», Salluste, Iug., 3, 3.

Il est important aussi de préciser, dès le début de notre travail, que la subordonnée d'exception (autant le type introduit par 'nisi', que le type introduit par 'quam') est doublement subordonnée: par rapport au verbe de la proposition régente et par rapport à un mot recteur, ou terme de référence (ou «antécédent») de la régente.

Une étape ultérieure est représentée par l'emploi des interrogations oratoires en tant que régentes à sens négatif. En voici des exemples:

- «Nunc quid mihi meliu st quam ilico hic opperiar erum, dum ueniat?», Plaute, Rud., 328.

- "Quod quom ita esse inuenero, quid restat nisi porro ut fiam miser?», Térence, Hec., 300.

Voir aussi Plaute, Men., 832-33 pour l'emploi de quam. En ce qui concerne la locution quam ut, celle-ci est assez fréquente chez Plaute (voir Aul., 76; Pseud., 1121; Rud. 220) ${ }^{2}$.

En fait, une proposition régente telle que: «quid mihi meliu'st ...?» (Plaute, Rud., 328) exprime preque le même sens que la régente suivante: »Quid restat ...? « (Térence, Hec., 300). La subordonnée introduite par quam (Plaute, Rud., 328) peut être considérée comme étant exceptive.

Cfr. Cicéron: «Quid iam restat? Quid habeo quod faciam pro tuis in me meritis, nisi ut eam fortunam quaecumque erit tua, ducam meam?», Mil., 100.

Quintilien: "Quid superest, nisi ut te puniri oporteat?», Inst., 4, 4, 4.

St. Avit: "Quid denique restat, // si mare transitur gressu, nisi nauibus arua // sulcentur caelumque suo decurrat ab axe // etc.?», 5, 623.

De telles formulations foisonnent dans le style élevé, mais aussi dans les monologues et les dialogues du latin familier (voir César, G., 7, 77, 15 - le discours de Critognate; Florus, 2, 2, 25 etc.).

D'ailleurs l'une des causes de la confusion existante à toutes les époques entre l'emploi de quam et celui de nisi réside précisément dans le type similaire et même identique de proposition régente ( $\left(N\right.$ Nihil aliud est ...» ${ }^{3}$.; ajoutons les régentes où les comparatifs ont valeur de superlatifs.)

Une autre cause de la confusion c'est que les subordonnées exceptives introduites par nisi sont, par essence, des subordonnées comparatives, et non pas des conditionnelles (voir sur cette question la discussion infra).

Il y a pourtant, en principe, une différence entre les subordonnées régies par nisi et celle introduites par quam, c'est-à-dire les propositions de nisi peuvent manquer sans 
que le sens de la régente en souffre, tandis que les propositions de quam sont nécessaires pour la compréhension de la régente. Cfr. la différence existante entre les relatives isolées et les relatives non-isolées.

Précisons aussi que le sens limitatif de nisi et de quam est quelquefois renforcé par des adverbes comme iam, nunc, tunc, denique, eo die, présents dans la régente (pour ce qui est de nunc, voir l'exemple cité - Plaute, Rud., 328; pour iam, voir Cicéron, Mil., 100; pour denique - St. Avit, 5, 623), ou comme modo tantum (= «seulement») qui étaient employés aussi bien dans la régente que dans la subordonnée. Des adjectifs tels que solus, unus, apparaissent dans la régente en tant que termes de référence (ou mots recteurs, ou bien "antécédents»), ou dans la subordonnée, pour rendre l'idée d'opposition et d'exclusion.

Voir une locution assez fréquente dans le latin populaire:

«tantum nisi quod», Pseudo-Quintilien, Decl., 14, 5, p. 269, $17^{4}$.

Voir un passage des écrivains chrétiens:

«quin etiam paternae clementiae uenia sola non sufficit, nisi et munera larga

multiplicet.», Ruricius, Ep., 1, 2, p. 354, 1. 15-17.

Les adverbes se rapportant à la quantité (en premier lieu tantum et tantumdem) acquièrent souvent, dans le latin populaire, un sens limitatif (voir Plaute, Pers., 517).

Une fois consolidé le sens limitatif d'exclusion de nisi (= «si ce n'est toutefois que», «à l'exception de»), la subordonnée d'exception est utilisée même en dépendance des régentes affirmatives. Pour ce qui est des subordonnées exceptives introduites par quam, leur existence est conditionnée par la présence des régentes à sens négatif.

Pour ce qui est de nisi et de ses locutions, en dépendance des régentes affirmatives, voici quelques occurrences:

- «Nam <e> quidem, nisi quod custodem habeo, liberum me esse arbitror.», Plaute, Capt., 394.

- Voir également Térence, H.T., 399-400, etc.

- Voir, à l'époque classique:

«Quam ob rem, si cadit in sapientem animi dolor -qui profecto cadit, nisi ex eius animo exstirpatam (esse) humanitatem arbitramur- quae causa est cur ....?», Cicéron, Lael., 48.

- Voir aussi César, G., 1, 5, 3, etc.

L'apparition, à l'époque préclassique, d'un assez grand nombre de locutions conjonctives fait augmenter l'emploi des subordonnées d'exception régies par des propositions affirmatives.

Les causes de l'apparition des locutions conjonctives sont de nature différente:

1. le souci de précision sémantique. Nisi exceptive pouvait être confondue avec nisi conditionnelle. Quam se confondait avec la conjonction comparative.

2. autant nisi que quam n'étaient pas adéquates pour indiquer l'exclusion de toute 
sorte de circonstances (de temps, d'espace, de manière, de but, etc.).

3. l'influence des locutions des propositions principales adversatives.

4. l'influence des locutions limitatives du grec ancien (telles que:

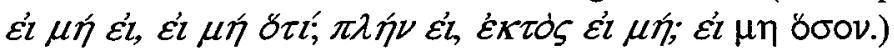

Les nouvelles ligatures exceptives seront acceptées dans les principaux registres latins. Nous en mentionnons les plus fréquentes à l'époque préclassique: nisi quod (Plaute, Capt., 394; ibid., 620-21; Térence, H.T., 643; ibid., 959 etc.); nisi quia (Plaute, Cist., 223-24; Truc., 786; Térence, Eun., 736, etc.); nisi ut (Plaute, Cist., 40-1; Térence, Eun., 74, etc.); nisi si (Caton, Agr., 138; Plaute, Curc., 51-2; Capt., 530; Térence, Ad., 594; Eun., 159-60, etc.); nisi qui (Plaute, Capt., 916; Trin., 439; Térence, An., 336-7, etc.).

En voici des exemples:

- "Quid uis, nisi uti maneat Phanium atque ex crimine hoc //

Antiphonem eripiam atque in me omnem iram deriuem senis?,

Térence, $\mathrm{Ph} .$, 322-3.

- "Tam a me pudica est quasi soror mea sit, nisi //

si est osculando quippiam inpudicior.», Plaute, Curc., 51-2.

D'autres locutions sont assez rares: nisi ubi, nisi cum ${ }^{5}$. D'un emploi assez rare sont aussi les locutions praeter quam quod (Térence, H.T., 399-400), praeter quam qui (Caton, Hist., 24; Térence, Eun., 77-8), extra quam si (créée sur le modèle de nisi sivoir Senatus Consultum de Bacchanalibus, dans C. I. L., I - 2, no. 581, 1, 296) et les locutions comparatives-exceptives: quam ut (plusieurs occurrences chez Plaute, citées déjà ), quam si (voir Plaute, Trin., 409-10; Térence, Eun., 62-3) et quam qui (Térence, Hec., 793).

Mentionnons que les locutions nisi quod/quia, praeter quam quod indiquent l'exclusion, ou l'isolation d'un fait par rapport au fait de la régente (= «excepté que»); nisi $u t$ est employé pour indiquer l'isolation d'un fait, mais aussi d'un but (= «excepté que»). Pour l'exclusion d'un but, voir Plaute:

- « .... Neque ego hanc superbiai //

Causa pepuli ad meretricium quaestum, nisi ut ne esurirem.», Cist., 40 41.

Les ligatures nisi si, quam si expriment l'exclusion d'une condition (= «excepté si»); nisi cum, nisi ubi - l'exclusion d'une période, ou d'un moment (= «excepté la période, le moment où»), nisi qui, quae, quod, praeter quam qui, quae, quod-l'exclusion d'une personne, ou d'un objet.

Les locutions nisi quod/quia, nisi si, nisi qui, praeter quam quod, praeter quam qui sont en général considérées par les spécialistes comme étant des pléonasmes. Selon nous, nisi est devenue de bonne heure un adverbe à sens exceptif; praeter (au sens: «à part», «sauf») introduisait également chez Plaute le complément d'exception. En voici des exemples: 
- «(uxor) quae nisi dotem omnia quae nolis habet.», Caecilius, Com., 144.

- «nullum praeter hunc diem.», Plaute, Merc., $585^{7}$.

Donc, les locutions renfermant nisi, énumérées antérieurement, contiennent en fait un adverbe exceptif et une conjonction de subordination.

En ce qui concerne les locutions formées à l'aide de praeter quam et de extra quam, celles-ci contiennent des pléonasmes. Le composé praeter quam est attesté chez les auteurs préclassiques en tant que subordonnant comparatif (voir Naevius, Colax, fr. 3, 34; Térence, H.T., $60^{8}$ ). Praeter quam devient assez tard adverbe limitatif introduisant le complément d'exception (voit César, $G ., 7,77,6$ etc.). Certains écrivains cultivés des époques classique, postclassique et tardive préfêrent employer praeter quod, praeter si, extra si, et non pas praeterquam quod, praeterquam si, extra quam si.

Dans certains cas, nisi si constitue également un pléonasme. Exempli gratia:

«Tibi ego numquam quicquam credam, nisi si accepto pignore.»

$$
\text { Plaute, Rud., 581, }
$$

En comparaison des formules correctes comme:

«deditionis nullam esse condicionem, nisi armis traditis.»,

César, G., 2, 32, 1.

\section{Toutes les locutions conjonctives de l'époque préclassique sont reprises par les} auteurs de l'époque classique.

Les locutions les plus fréquentes sont: nisi quod, praeter quam quod et nisi qui. Pour ce qui est de nisi quod, voir Cicéron, Fin., 4, 28; Fam., 13, 1; Salluste, Iug., 95, 3 etc. ${ }^{9}$; pour praeter quam quod, voir Cicéron, $H$. resp., 41; Cicéron, Att., 9, 15,5; ibid., 15, 15, 2, etc. ${ }^{10}$; pour nisi qui, voir Cicéron, Ph., 2, 81; Brut., 61; César, G., 1 , 30, 5; Salluste, Iug., 17, 6; ibid., 75, 3, etc.

Praeter quam qui est attestée surtout dans Bellum Hispaniense - voir 22, 1; ibid., 24; ibid., 34; voir aussi César, G., 1, 5, 3 11; Népos, 25, 21.

En ce qui concerne nisi quia, celle-ci était considérée spécifique du latin populai$\mathrm{re}^{12}$ et n'apparaît aucunement chez les auteurs classiques.

Ajoutons que Cicéron évite l'emploi de nisi quod et de praeter quam quod dans ses discours; chez César, ces deux locutions sont inexistantes ${ }^{13}$. Nisi si est rare chez les grands écrivains classiques (un seul exemple chez César, cité antérieurement - G., 1, $31,14)$. Disons aussi que César préfêre construire nisi avec l'infinitif, plutôt que d'employer nisi ut suivie du subjonctif (voir $G ., 7,77,15$ ).

Pour l'exclusion d'une condition, Cicéron et Salluste choisissent d'habitude nisi, nisi forte, nisi uero (voir Cicéron, Lael., 48; Verr., 2, 3, 149 etc.; Salluste, Iug., 3, 3). Rarement, nisi indique chez Cicéron l'isolation d'un fait (Rosc. Am., 108).

César emploie nisi autant pour l'exclusion d'une condition (voir $G ., 1,22,3 ; C$., 3, 44,1 ) que pour l'exclusion d'un fait (voir G., 5, 13, 4; ibid., 7, 77, 15). Nisi pour l'exclusion d'un fait est également attestée à l'époque préclassique (voir Térence, Eun., 735-6) et même dans Bellum Africum $(16,4)$. 
Nisi si fait pourtant son apparition chez Cicéron (voir Ph., 2, 70; Fam., 14, 2, 1 et d'autres passages ${ }^{14}$ ), Népos $(25,13)$, même chez Varron (L.L., 6, 29).

Nisi ut est fréquente chez Cicéron (voir Mil., 100; Vat., ${ }^{15}$ ); un seul exemple chez César $(C ., 1,63,2)$, un seul exemple dans Bellum Africum $(69,5) .{ }^{16}$

La locution nisi cum est beaucoup employée par les classiques, voir Varron, L.L., 5, 165; Cicéron, Ph. 11, 16, etc.; César, G., 6, 18, 3; Salluste, Iug., 44, $4^{17}$.

Les propositions comparatives-exceptives sont généralement introduites chez les auteurs classiques par quam suivie de l'indicatif, de l'»Accusativus cum Infinitivo», de l'infinitif «seul», ou du subjonctif paratactique (voir Cicéron, Verr., 2, 2, 58; Lael., 79; Brut., 219; César, C., 2, 32, 4; Hirtius, G., 8, 49, 2, etc.). Parfois on emploie quam quod suivie de 1'indicatif (voir Cicéron, Sest., 2; Brut., 218). Quam ut est rarement attestée chez les écrivains classiques (Cicéron, Lig., 38; Rab. perd., 2, 4; Népos, 6,1; César, $C$., $2,32,4)$.

On décèle aussi dans les textes des auteurs classiques la locution quam si, voir Salluste:

«neque se aliter tutum putet quam si intestabilior metu uestr fuerit.»,

$$
\text { Hist., 1, 55, } 1 .
$$

Chez Cicéron on rencontre aussi la locution à sens essentiellement exceptif extra quam si (Inu., 2, 172; Rep., 1, 10); toujours chez Cicéron on découvre extra quam qui (Tusc., 1,17$)^{18}$. La locution extra quam si semble avoir été agréée par les juristes, à cause de sa précision sémantique: dans certains passages, Cicéron cite les mots du jurisconsulte Q. Mucius Scaeuola (consul en 95 av. Chr.) - voir Cicéron, Inu., 1, 56; Att., 6, 1, 15.

Mentionnons aussi les locutions récemment apparues à l'époque classique:

- nisi posteaquam, chez Cicéron, Ac. 2, 69.

- praeter si, attestée chez Varron, R. r., 1, 41, 5; la locution a été reprise par Oribase, Syn., 5, 49 et par d'autres auteurs tardifs ${ }^{19}$.

- praeter quom, voir Varron, L. L., 7, $105^{20}$ (Varron emploie également nisi cumvoir la discussion antérieure).

- praeter qui - Cicéron, Att., 5, 3, 22l.

Importante est la locution 'tantum quod', attestée pour la première fois chez Cicéron:

«componit edictum iis uerbis, ut quiuis intellegere possit unius hominis causa conscriptum esse, tantum quod hominem non nominat.», Verr., 2, 1, 116.

Voir également Cicéron, Verr., 2, 3, 124.

Acceptée dans le latin populaire, tantum quod réapparaît chez Tite-Live, 33, 4, 6; Pétrone, 76, 11; cette locution est ensuite attestée chez Apulée, D. Socr., 8, 46, Solinus, 19, 19; Tertullien, Nat., 1, 4, (p. 64, 13) et d'autres auteurs de la basse époque ${ }^{22}$.

Présentant un haut degré de précision sémantique, ces nouvelles ligatures exceptives sont entrées en concurrence avec les vieilles locutions: praeter si concurrençait nisi 
si et extra quam si; tantum quod - nisi quod. Quoique souvent attestées dans les siècles suivants, nisi si et nisi quod commencèrent peu à peu à être marquées par une certaine ambiguité et finirent par être remplacées par «si-non (quod)», "excepto quod», «excepto si», de même que par les locutions formées à l'aide des adverbes 'praeter' et 'tantum' (voir la discussion infra).

Notons que nisi, en tant qu'adverbe limitatif d'exclusion, est utilisé à partir de l'époque préclassique pour introduire le complément d'exception (voir l'exemple cité - Caecilius, Comm., 144).

A l'époque classique, nisi est, vraisemblablement, le plus fréquent introductif du complément d'exception, souvent dans une proposition négative au point de vue du sens et de la forme, ou dans une interrogation oratoire (proposition négative au point de vue du sens). Exempli gratia:

«Quid enim unquam domus illa uiderat nisi pudicum, quid nisi ex optimo more et sanctissima disciplina?», Cicéron, Ph., 2, 69.

Précisons que le complément d'exception est lui aussi doublement subordonné: par rapport au verbe de sa proposition et par rapport à un terme de référence de la même proposition. Dans le passage de Philippicae que nous venons de citer, le complément d'exception est subordonné par rapport au verbe (uiderat), mais également il se rapporte au pronom interrogatif quid.

Pour ce qui est du complément d'exception introduit par nisi, voir aussi Cicéron, Lael., 18; ibid., 20; ibid., 65; Mil., 104; Att., 4, 3, 2 etc.; César, G., 5, 41, 5; Salluste, Cat., 13, 1, etc.

Pour introduire le complément d'exception, on emploie aussi praeter, en tant qu'adverbe et préposition, de même que praeter quam - adverbe. Extra, en tant que préposition gouvernant l'Accusatif, est assez rare aux époques préclassique et classique $^{23}$. Par exemple:

«ceterae multitudini diem statuit, ante quam sine fraude liceret ab armis

discedere, praeter rerum capitalium condemnatis.», Salluste, Cat., 36, 2.

«Atque ego hanc sententiam probarem, ...., si nullam praeter quam uitae nostrae iacturam fieri uiderem.», César, G., 7, 77, 6.

Assez fréquent est le complément comparatif-limitatif introduit par quam: «cuius ex omni uita nihil est honestius quam quod cum mima fecit diuortium.», Cicéron, Ph., 2, 69.

Soulignons que l'existence du complément introduit par 'quam' est conditionnée par la présence du verbe négatif dans la même proposition, tandis que nisi, praeter, extra, praeter quam introduisent des compléments d'exception aussi bien dans des propositions négatives que dans des propositions affirmatives.

L'emploi de différents adverbes pour introduire le complément d'exception nous indique une liaison faible entre le mot recteur et le complément et, en outre, l'existence d'une relation comparative entre les deux termes. Grâce à l'idée de comparaison, le complément d'exception se trouve souvent au même cas que le terme de référence, par 
exemple: «Est enim amicitia nihil aliud nisi omnium diuinarum humanarumque .... consensio.», Cicéron, Lael., 20.

De plus on peut parler d'un processus d'unification des mots introductifs du complément d'exception et de la subordonnée correspondante, processus qui s'accentue dans le latin populaire de la basse époque.

Ajoutons que chez Cicéron l'idée de limitation est parfois soulignée par le fait que le grand orateur ajoute à l'adverbe exceptif nisi l'Ablatif du substantif exceptio précédé de la préposition cum:

«Homines mortales necesse est interire sine adiunctione; ut cibo utantur, non necesse est, nisi cum illa exceptione: extra quam si nolint fame perire.», Inu., 2, $172^{24}$.

Pour ce qui est de la formule «cum exceptione», voir aussi Cicéron, Ep. Ad Quint., $1,1,37$.

Nous devons y ajouter que cette modalité d'expression du complément d'exception reste le privilège des auteurs cultivés. Exempli gratia: «sub hac exceptione», Pline, Ep., 1, 2, $5^{25}$.

En revenant à la subordonnée d'exception, nous tenons à résumer les conditions sous lesquelles celle-ci est employée:

- la proposition d'exception est doublement subordonnée; tout d'abord elle se trouve en dépendance du verbe de la régente; en second lieu, elle se rapporte à un terme de référence (mot recteur, "antécédent») de sa principale.

- le verbe régent est souvent accompagné de non, nec, haud, «ne-quidem». Si l'on n'emploie pas ces négations, on trouve dans la principale des pronoms, des adjectifs ou des adverbes tels que: nemo, nequis, nullus, nihil, numquam, nusquam etc.

- l'exclusion est conçue le plus souvent par rapport à la sphère sémantique du sujet, ou de l'attribut. L'isolation s'opère aussi par rapport au complément d'objet direct, ou au complément d'objet indirect, ou bien par rapport aux compléments circonstantiels (surtout ceux indiquant la manière, le temps et l'espace). L'exception vise aussi le complément sociatif, le complément d'accompagnement, le complément d'agent et le complément de nom.

La proposition régente prend souvent la forme:

«nihil aliud est (fuit) nisi ....», ou:

«nihil fecit nisi ...».

Pour ce qui est de la formule «nihil aliud agitur quam ut ...», voir Cicéron, $R a b$. perd., 2, 4.

Souvent les propositions régentes sont des interrogations oratoires comme par exemple: «Quid mihi restat nisi ...?».

L'existence des comparatives-exceptives est conditionnée par la présence des régentes à sens négatif. 
Si la proposition régente est affirmative, celle-ci renferme en qualité de termes de référence des noms communs et des noms propres, des pronoms, des adjectifs tels que: Pompeiani, uos, multi, reliqui, omnes, quisquis etc. Par exemple:

«Quod exspectaui, iam sum adsecutus, ut uos omnes factam esse aperte coniurationem contra rem publicam uideretis: nisi uero si quis est qui Catilinae similes cum Catilina sentire non putet.», Cicéron, Cat., 2, $6^{26}$.

Voici un passage où l'exception s'opère par rapport à un attribut:

"Nam <e>quidem, nisi quod custodem habeo, liberum me esse arbitror.», Plaute, Capt., 394.

A voir également un passage où le terme de référence est un complément de nom: «.... circumueniunt urbem Vspen, editam loco et moenibus ac fossis munitam, nisi quod moenia non saxo, sed cratibus et uimentis ac media humo .... inualida erant.», Tacite, Ann., 12, 16, 2.

- Il y a certains corrélatifs dont le sens est: «encore», «en plus», «finalement», tels que: iam, nunc, denique (voir la discussion supra).

D'autres corrélatifs sont des adverbes ayant le sens: «seulement» (par ex.: tantum).

- La différence concernant l'emploi de quam et de nisi s'estompait souvent. Même Cicéron emploie quelquefois nisi à la place de quam, en relation avec un adverbe impliquant la comparaison.

Exempli gratia: «nec mihi aliter potuisse uideor ... consilia frangere, nisi... coniunxissem.», Fam., 1, 9, 21.

Voir également Bellum Africum, 16, 4; Quintilien, Inst., 4, 2, 66. A l'époque tardive, les occurrences de nisi au lieu de quam sont nombreuses, par exemple: Ruricius l'Evêque, Ep., 2, 17, p. 402, 1. 12-13 (voir la discussion infra).

Par contre, quam apparaît quelquefois à la place de nisi, dans des passages qui ne contenaient pas de mots présupposant la comparaison. Exempli gratia:

«Quid est dei uoluntas quam dei sapientia?», Faustinus, Trin. 1, 14 (p. 49 C) ${ }^{27}$

- On observe, à partir de l'époque préclassique, que certaines locutions exceptives entrent en concurrence les unes avec les autres. C'est ainsi que nisi quilquae/quod - très précise - est parfois préférée à la locution nisi si, par exemple:

«oleum ne tangito, nisi quod custos dederit.», Caton, Agr., 145, 2.

D'autre part, nisi si remplace parfois nisi cum, cette dernière - très concrète: «numquam poëtor, nisi si podager (sum).», Ennius, Sat., 3, 2.

Dans le passage d'Ennius, le terme de référence est numquam (= jamais).

Cfr. César: «In reliquis uitae institutis, hoc fere ab reliquis differunt, quod suos liberos, nisi cum adoleuerunt .... palam ad se adire (s.-en.: puerili aetate) non patiuntur.», G., 6, 18, 3 .

Cfr. aussi Tite-Live:

«hic noster, hic plebis nostrae habitus fuit eritque semper, nisi si quando a uobis proque uobis arma acceperimus.», 6, 26, 5 . 
Nisi si est parfois employée de manière erronée à la place de nisi conditionnelle (résultat de la confusion entre les subordonnées exceptives et les conditionnelles négatives). En témoigne ce passage:

«Edim, nisi si ille uotet ....», Plaute, Trin., 474.

Le terme de référence peut manquer, voir César, $G ., 6,18,3$ (passage cité).

Quelle est la définition de la subordonnée d'exception?

- Sur le plan sémantique, la subordonnée d'exception «indique une circonstance particulière qui, ajoutée à la principale, lui en restreint la portée.» ${ }^{28}$

- la subordonnée d'exception est, en dernière analyse, une comparative d'exclusion, ou d'isolation. Quoique empruntée à la proposition conditionnelle, nisi indique la comparaison exceptive. Pour ce qui est de quam, celle-ci est spécifique de la subordonnée comparative. Les arguments concernant la nature comparative des propositions d'exception sont nombreux:

1. la relation sémantique entre la régente et la subordonnée;

2. la faible liaison entre la régente et la subordonnée;

3. la confusion permanente entre l'emploi de nisi et celui de quam. Outre cela, praeter quam est confondue avec praeter quam quod et praeter quam si; extra quam - avec extra quam si, et ainsi de suite.

4. Les subordonnées exceptives régies autant par nisi que par quam imitent souvent la construction des vraies comparatives. En dépendance d'une régente construite avec l'infinitif «seul», dans la subordonnée exceptive on emploie également l'infinitif «seul». Par exemple:

«... neque amplius facere nisi hostium iacula uitare.», Bellum Africum, 16, 4.

Cfr. César, G., 7, 1, 8. A voir également Commodien, $A p$., 687-88.

5. formation des locutions exceptives à l'aide de praeter et tantum limitatifscomparatifs.

- de l'idée d'isolation résulte l'idée d'opposition de la subordonnée et certaines similitudes avec les principales adversatives. L'opposition logique est parfois marquée, dans la subordonnée, par des adverbes tels que uero, tamen, forte et même par forsitan ${ }^{29}$, juxtaposés à la conjonction, ou à la locution limitatives. Parfois on rencontre aussi quidem - voir Plaute, Mil., 182-84.

Certaines occurrences de nisi et de nisi tamen, nisi quia tamen sont même interprétées par les grammairiens comme étant «rein adversative» ${ }^{30}$.

- Les subordonnées d'exception renferment parfois une nuance d'addition, voir Plaute, Truc., 786, etc.

- Le mode couramment employé dans les subordonnées d'exception c'est l'indicatif. Précisons qu'il s'agit d'habitude de l'indicatif de la réalité, parfois de l'indicatif de l'éventualité. Quelquefois, on emploie l'optatif, pour indiquer la possibilité et l'irréalité; l'optatif apparaît également chez les auteurs cultivés dans l'oratio obliqua (le discours indirect). On rencontre aussi le subjonctif proprement dit, pour expri- 
mer l'idée finale. L'infinitif et même l'»Accusatiuus cum Infinitiuo» apparaissent après nisi et quam seules (et non pas dans le cas des locutions conjonctives).

- A l'époque préclassique se constituent certaines structures grammaticales et lexicales qui se transmettent aux siècles suivants. Le terme de référence est obligatoire pour l'existence de la subordonnée d'exception ${ }^{31}$. Des locutions précises apparaissent, indiquant l'exclusion d'une condition, d'un but, etc. L'indicatif de la réalité est beaucoup employé. Dans la régente se trouvent parfois des corrélatifs. Les règles du latin cultivé imposent l'emploi de nisi devant l'Ablatiuus absolutus et le Participium coniunctum à sens exceptif.

- Etant donné que la subordonnée d'exception est doublement subordonnée et vu qu'elle peut manquer sans que le sens de la régente soit endommagé, cette subordonnée est d'habitude placée après sa principale. Rarement, à cause d'une emphase oratoire hors du commun, ou pour des raisons prosodiques, la subordonnée d'exception occupe la première place (voir Plaute, Capt., 394; César, G., 1, 31, 14).

- Les signes de ponctuation reflètent cette faible dépendance de la subordonnée d'exception. Voir une citation de Cicéron: «mors quidem illata per scelus isdem et poenis teneatur et legibus. Nisi forte magis erit parricida, si quis consularem patrem, quam si quis humilem necarit ...», Cicéron, Mil., 17.

Le plus souvent la subordonnée d'exception est confondue avec la subordonnée conditionnelle.

Les causes de cette confusion sont de nature différente:

- l'origine comparative de ces catégories de subordonnées;

- l'emploi de l'indicatif de l'éventualité et de l'optatif autant dans la conditionnelle que dans la proposition d'exception;

- l'emploi de nisi dans ces deux catégories de subordonnées;

- en ce qui concerne les subordonnées d'exception introduites par des locutions, celles exprimant l'isolation d'une condition comptent parmi les plus fréquentes (des subordonnées régies par nisi si, extra quam si, praeter si, praeter quam si et, enfin, par la locution quam si).

Etant donné la confusion entre les subordonnées conditionnelles et celles d'exception, nous tâcherons de donner aussi la définition des subordonnées conditionnelles (appelées aussi protases, hypothétiques, ou conditionnantes):

- la subordonnée conditionnelle est un développement ancien des propositions comparatives ${ }^{32}$.

- Du fait que la subordonnée conditionnelle indique la condition de l'accomplissement de l'action (ou de l'état) de la régente, celle-ci est étroitement liée à sa principale. Les modalités de rendre en latin le complément de condition sont également éloquentes pour l'importance de la condition en rapport avec le verbe recteur. On emploie souvent la préposition sub (voir: «sub condicione», «sub condicionibus»Tite-Live, 6, 40, 8; 21, 12, 4; «sub lege», «sub nomine», «sub poena» etc.); dans une moindre mesure, on emploie la préposition in (par exemple: «in discordia ... 
pax ciuilis esse non potest.», Cicéron, $P h ., 7,23)$, ou l'Instrumental non-prépositionnel (par ex.: «iubere eï praemium tribui, sed ea condicione ne quid postea scriberet.», Cicéron, Arch., 25).

- Les spécialistes affirment que l'idée exprimée dans la conditionnelle, le mode et le temps employés dans la protase déterminent en grande mesure le choix du mode et du temps dans la principale, et non pas inversement. ${ }^{33}$

- Vu que l'action de la régente ne saurait se réaliser que sous la condition de l'accomplissement de l'action de la subordonnée, vu également l'origine et le sens des conjonctions de la subordonnée conditionnelle, en y ajoutant la discussion sur l'emploi des locutions formées avec modo, tantum, quidem, l'apparition de certains corrélatifs, modes et temps dans la principale, la subordonnée conditionnelle peut être appréciée comme étant «une comparative restrictive».

- Les modes employés d'habitude dans la conditionnelle sont: l'indicatif à valeur d'optatif et l'optatif. Le subjonctif et, rarement, l'optatif apparaissent dans les conditionnelles introduites par dum, dum modo et modo au sens «pourvu que».

- Dès leur naissance (en indo-européen), la position normale pour les subordonnées conditionnelles c'est d'être placées avant leurs régentes.

\section{L'EXPRESSION DE L'IDEE D'EXCEPTION AUX EPOQUES POSTCLASSIQUE ET TARDIVE.}

A. Les écrivains cultivés des époques postclassique et tardive continuent d'employer les conjonctions simples 'nisi' et 'quam' pour introduire les subordonnées d'exception, quoique les locutions eussent l'avantage d'une haute précision sémantique. On rencontre aussi, chez les auteurs cultivés, nisi uero et nisi forte.

Pour l'emploi de nisi, voir St. Avit:

«Quid denique restat, .... nisi nauibus arua sulcentur ....?», 5, 623.

Quintilien, après une proposition régente du même type, emploie la locution nisi ut (voir Inst., 4, 4, 4).

Pour ce qui est de l'emploi de quam, voir Tite-Live, 4. 26, 12. Voir aussi une citation de Jordanès, auteur du VI ${ }^{\mathrm{e}}$ siècle:

"... quid aliud quam uictor de uictoribus atque etiam, quia Chartago ${ }^{34}$ non

cesserat, de fortuna triumphauit?», Rom., 172.

La confusion entre l'emploi de nisi et celui de quam continue à exister. Voici un passage où nisi apparaît à la place de quam si:

«... ad quam (ciuitatem) tamen aliter peruenire non possumus, nisi caritatis gradibus conscendamus ....», Ruricius l'Evêque, Ep., 2, 17 (p. 402, 1.12-13).

B. Un phénomène important c'est le remplacement de 'nisi', de 'nisi si' et même de 'nisi quod' par 'si-non', phénomène attesté chez les auteurs qui reproduisaient le latin populaire, ou qui en étaient influencés sans le vouloir. Par exemple: 
- «Futura erat indubitanter casta et sincera generatio, si non intercessisset inimica transgressio.», Faustus Reiensis, Grat., 1, 2 (p. 14, 1. 9-10);

- «inhonestus, cupidus et cruentus est cuiuslibet facinoris seruus, si peccatis non reluctetur.», Faustus Reiensis, Grat., 1, 17 (p. 54, 1. 7-8).

La tendance d'exprimer l'idée d'exception à l'aide de «si - non» est moins évidente à l'époque postclassique (voir Tite-Live, 3, 67, 2; idem, 3, 67,5; Pétrone, 140, 4). Importantes dans le processus de glissement des conditionnelles vers les subordonnées d'exception ce sont l'idée d'isolation de la condition et l'idée d'opposition entre le prédicat de la subordonnée et celui de la régente (opposition marquée par non et des adverbes tels que: tamen, quidem, tantum). Plus tard, au IIe siècle p. Chr., on y ajoute le placement de la subordonnée après sa principale (voir Terentianus Maurus, 690 ${ }^{35}$ ). Nous désirons souligner que «si-non» peut exprimer l'isolation d'une condition ou d'un fait. 'Si non' est aussi un introductif pour le complément d'exception.

C. L'apparition des hyperurbanismes:

1. L'emploi de nisi quis au lieu de si quis non. A voir une citation de l'Evangile de Iohannes:

«nisi quis renatus fuerit ex aqua et spiritu sancto, non potest intrare in regnum dei.», apud Faustus Reiensis, Spir. Sanct., 2, 4 (p. 144, 1. 15-16).

Voir également Faustus Reiensis, Spir. Sanct., 2, 5 (p.144, 1. 23-4); ibid., 2, 5 (p. 145, 1. 2-3), etc.

2. La construction de nisi et de quam avec l'»Accusatiuus cum Inf.», à la place de l'indicatif, ou du subjonctif. Exempli gratia:

«... quid aliud demonstrauit, nisi se gubernasse uiuentem quem non dereliquit exanimem?», Faustus Reiensis, Serm., 13 (p. 276, 1. 5-7).

Pour ce qui est de quam accompagnée de l'»Accusatiuus cum Inf.», voir en premier lieu Tite-Live, 4, 26, 12; voir ensuite Ennode, Ep., 3, 18 (p. 85, 1. 14-15).

Cfr. Hirtius: «Nihil enim minus uolebat quam sub decessu suo necessitatem sibi

aliquam imponi belli gerendi ... «, G., 8, 49, 2.

Chez Ennode on rencontre aussi la locution nisi cum suivie de l'»Accusatiuus cum Inf.» - voir Lib. pro synodo, p. 288, 1. 12-15.

D. Les locutions attestées à l'époque classique se retrouvent chez les auteurs postclassiques.

Nisi quod est employée par Tacite, Ann., 1, 33, 3; ibid. 6, 24, 1; ibid., 11, 24, 4, etc.

Pour nisi ut, voir Quintilien, Inst., 4, 4, 4; ibid., 5, 10, 57 etc.; Tacite, Dial., 33, 5; Velleius Paterculus, 2, 17, 1; Suétone, Cal., 23, $2^{36}$.

Nisi si apparaît chez Tite-Live, 6, 26, 5; Columelle, Vitruve, Tacite et d'autres. Nisi cum est attesté chez Ovide, Pont., 3, 6, 57. Pour ce qui est de la locution nisi si quando, voir la discussion infra sur les pléonasmes.

Nisi qui est employée par Tacite, Ann., 2, 24, 2; ibid., 16, 18, 2 et d'autres écrivains. 
Praeter quod se substitue à la locution praeterquam quod, voir Apulée, Met., 4, 27. Praeter quod devient ensuite fréquente chez les auteurs tardifs (voir Lactance, 3, 8, 13; Opif., 19, 637; voir également Jordanès, Get., 106).

Praeter qui remplace praeterquam qui - voir Florus, 2, 6, 24; Jordanès, Rom., 192, etc.

Les locutions formées à l'aide de l'adverbe praeterquam sont rares à l'époque postclassique. Pour ce qui est de praeterquam quod, voir Tite-Live, 1, 55, 8; idem 21, 10 , 1; idem, 22, 38, 12; pour praeterquam ut, voir Tite-Live, 4, 4, 12; pour praeterquam $s i$, voir Ulpien, Dig., 21, 1, 12, 3; praeterquam ubi-Pline, Nat., 5, 65; pour praeterquam qui - Tite-Live, 23, 31, 2; Vitruve, 10, 11, $5^{38}$.

Les locutions formées sur extraquam sont également rares. Extraquam si apparaît chez Tite-Live, 38, 38, 9: idem, 39, 18, 7; pour extraquam qui, voir Tite-Live, 26, 34, $6{ }^{39}$.

La locution tantum quod, attestée pour la première fois chez Cicéron à valeur exceptive, est beaucoup employée, autant chez les écrivains cultivés (Tite-Live, 33, 4, 6; Apulée, D. Socr., 8, 46), que chez les auteurs qui reproduisaient le langage populaire (voir Pétrone, 76, 11 - le discours de Trimalchion). Cette locution réapparaît chez les auteurs tardifs 40 .

Quam ut est attestée chez Suétone, Aug., 83; Tib., 24; ibid., 32, etc.; quam si apparaît chez Columelle, $R . r ., 4,2$, etc.

E. Les écrivains cultivés de l'époque postclassique forment de nouvelles locutions limitatives. Ces locutions, fondées sur l'idée de comparaison, sont des formations savantes, peu employées. Exempli gratia:

- «supra quam quod» - deux exemples chez Tite-Live (22, 3, 14; ibid., 27, 20, 10);

- «super id quod» - un seul exemple chez Tacite $(A n n ., 4,11,1)^{41}$.

Parfois on trouve dans les textes des agglomérations d'adverbes à fonction différente. Voici un passage de Tite-Live:

«Senatum uero incitare aduersus legem haud desistebat: ne aliter descenderent in forum, cum dies ferendae legis uenisset, quam ut qui meminissent sibi pro aris focisque et deum templis ac solo ... dimicandum fore.», 5, 30, 1 .

Comme on l'observe, il y a une locution quam ut qui dans laquelle quam présente un sens comparatif-exceptif, tandis que $u t$ est un adverbe qualificatif (ou caractérisant), mettant en relief le pronom relatif ${ }^{42}$.

F. Importantes sont, à l'époque postclassique et à l'époque tardive, les nouvelles locutions formées sur l'adverbe 'excepto'. Aux époques archaïque et préclassique, exceptus était un participe parfait, utilisé parfois en tant que verbe de l'Ablatif Absolu. Devenu ensuite adverbe, excepto introduisait le complément d'exception et servait à la formation des locutions conjonctives.

La plus fréquente locution de excepto, attestée pour la première fois au temps d'Auguste, c'est excepto quod. Voir Horace: 
«Haec tibi dictabam ...., //

excepto quod non simul esses, cetera laetus.», Ep., 1, 10, v. 49-50.

Ovide emploie également excepto quod - voir Pont., 4, 14, 3; Trist., 3, 6, 12. On retrouve cette locution chez Quintilien, voir Inst., 9, 4, 79, Pline le Jeune, Ep., 8, 1, 1 et chez d'autres auteurs postclassiques ${ }^{43}$. La locution excepto quod est fréquente à l'époque tardive (voir Donat, Ter. - Ad., 380; Vulgata, Gen., 9, 4; St. Benoît, Reg., 10 etc. ${ }^{44}$ ).

La locution excepto si, moins fréquente chez les écrivains postclassiques, fait souvent son apparition à l'époque tardive (voir Peregrinatio Aetheriae, 27, 5; St. Grégoire le Grand, $E p ., 5,15$, etc.) ${ }^{45}$. Excepto cum est rarement attestée (trois exemples dans le Thesaurus linguae Latinae: le Grammairien Virgilius, Epit., 3, p. 11, 9; idem, Epist., 1, p. 118, 12; Beda, Gramm., 7, 236, 2446).

Les écrivains cultivés emploient parfois la structure de l'Ablatif Absolu suivie d'une proposition à fonction d'apposition, introduite par quod, ou par si. Chez Sénèque l'Orateur, on trouve «excepto eo quod ...» (Contr., 2, 2, 9); chez le jurisconsulte Paulus - «excepto eo si ...» (Dig., 46, 2, 10).

L'idée d'exception est parfois rendue par des Ablatifs Absolus correctement construits, par exemple:

«Exceptis enim Latinis, hanc (s.-en.: litteram $Q$ ) nulla alia lingua habet.»,

St. Isidore, Et., 1, 4, 13.

Ce sont des imitations des formulations d'Ablatifs Absolus fréquentes chez Cicéron, du type suivant:

«qua (amicitia) quidem haud scio an, excepta sapientia, quicquam melius homini sit a dis immortalibus datum.», Lael., 20.

Voir également Cicéron, Lael., 104, etc.

Ajoutons que le participe excepta (ou exceptis) renferme (en soi-même) le sens limitatif ; c'est pourquoi l'adverbe exceptif nisi ne précède pas de telles formulations. Cfr. César: «deditionis nullam esse condicionem, nisi armis traditis.», G., 2, 32, 1.

G. Une particularité commune aux auteurs influencés par le latin populaire et aux auteurs cultivés des époques postclassique et tardive c'est l'emploi des locutions amples contenant des pléonasmes. En voici quelques exemples - en ordre chronologique:

- «nisi si quando», chez Tite-Live, 6, 26, 5 (passage cité);

- «praeter quam si quae Macedoniam peterent, omnes ... spoliabat naues.», Tite-Live, 44, 29, 4.

- «nec quicquam aliud in hac epulatione captabant, nisi tantum ne esurirent.», Pétrone, 141, 10.

- «tantum nisi quod», Pseudo-Quintilien, Decl., 14, 5, p. 269, 1. 17.

- «solum nisi quia», Filastrius, 36, 147.

On observe dans ces passages soit l'accumulation de conjonctions de subordination («si - quando»; «si -qui»), soit l'agglomération d'adverbes limitatifs («tantum nisi», «solum nisi»). 
H. En ce qui concerne l'inventaire de locutions à la basse époque, outre l'emploi des pléonasmes, on constate la réapparition de la locution nisi quia, évitée par les écrivains de l'époque classique et de l'époque postclassique. Nisi quia, exactement comme nisi quod, a le sens de «excepté que».

Nisi quia est attestée d'habitude chez les ecclésiastiques, voir Cassien, Con., 3, 7, 8; Faustus Reiensis, Serm., 30, p. 341, 1. 8; Ennode, Lib. pro synodo, p. 296, 1. 1-3; St. Avit, p. 25,8 etc. Voir également l'exemple cité - Filastrius, 36,1 . Nisi quia est également employée dans Itala et Vulgata, à côté de nisi quod ${ }^{48}$.

Certains auteurs tardifs emploient nisi quia de manière erronée, à la place de nisi conditionnelle (confusion entre les subordonnées d'exception et les conditionnelles), par exemple:

«neque .... Danihel leones et tres pueri uincerent ignes, nisi quia credentes fuissent.», Lucifer l'Evêque, Athan., 1, 41 (p. 140, 1. 17).

Voir également Faustus Reiensis, Serm., 29 (p. 338, 1. 20-21).

A l'époque tardive on rencontre aussi prater quia au sens de praeter quod (voir Oribase, Syn., 6, $16^{49}$ ).

Une tendance importante dans le latin populaire, de même que dans le latin cultivé, c'est l'extension des locutions fondées sur 'nisi', 'praeter' et 'tantum', au détriment des locutions formées à l'aide de 'praeterquam' et 'extraquam'.

Les plus fréquentes sont les locutions fondées sur 'nisi'. Pour ce qui est de nisi quod, voir Tertullien, Anim., 34, 5; Priscillien, Tract., 10, 140; Faustus de Riez, Serm., 13 (p. 276, 1. 12-3); Salvien, Eccl., 3, 89; Gub. Dei, 4, 47; Jordanès, Rom., 266; ibid., 269; ibid., 307; Get., 122, etc. ${ }^{50}$.

Plusieurs spécialistes, et des meilleurs (Wilh. Meyer-Lübke, Fr. Stolz, J. G. Schmalz, J. B. Hofmann, A. Szantyr) affirment que nisi quod et nisi quia ont survécu en portugais (voir nego et nega) ${ }^{51}$.

Nisi ut est aussi fréquente à l'époque tardive, voir Priscillien, Tract., 10, 141; Ennode, Ep., 3, 17 (p. 85, 1. 1-3); Victor Vitensis, Hist. persec. Afr. prou., 3, 46; Jordanès, Rom., 110, etc. Pour nisi si, voir Tertullien, Anim., 46, 10; Optatianus Porphyrius, Sent. C., 35; Mulomedicina Chironis, 207, etc.

Moins fréquentes sont: nisi cum (voir Ennode, Ep., 2, 7, p. 46, 1. 9-10; Lib. pro synodo, p. 288, 1. 12-15) et nisi postquam (Ennode, Ep., 1, 7, p. 16, 1.22-3).

Quant à nisi qui, celle-ci est beaucoup employée à la basse époque: Claudien Mamert, Stat. An., 1, 24 (p. 84, 1. 17); Ennode, Lib. pro synodo, p. 291, 1.17 sqq; Victor Vitensis, Hist. persec. Afr. prou., 3, 47; Jordanès, Get., 189, etc.

Praeter si est également employée à l'époque tardive - voir Oribase, Syn., 5, 49 etc. ${ }^{52}$. Pour ce qui est de praeter quod, praeter quia, praeter qui, voir la discussion supra.

La locution tantum quod est attestée chez Solinus, 19, 19 et Tertullien, Nat., 1, 4453. Tantum apparaît souvent juxtaposé à la conjonction nisi (voir Commodien, Ap., 687-8), ou à l'adverbe exceptif nisi (créant, dans cette dernière situation, des pléonasmes - voir la discussion point $\mathrm{G})$. 
La catégorie des comparatives-limitatives continue à exister. A part l'emploi de quam seule, il y a de nombreuses attestations de locutions fondées sur quam, telles que: quam quod, quam ut, quam si, quam qui. Par exemple:

«Nihil maius poterit efficere quam si ipse ad sanitatem propriam meruerit peruenire.», Faustus Reiensis, Serm., 29 (p. 340, 1. 4-5).

Pour ce qui est de quam qui, voir le passage suivant:

«.... quia nihil est homini melius quam quod ipse uult.», Faustus Reiensis, Serm., 29 (p. 339, 1. 20-21).

Pour quam qui, voir aussi Lucifer l'Evêque, Athan., 1, 22 (p. 105, 1. 10), etc.

Nisi quod remplace parfois quam quod, par exemple:

«... natio saeua .... uenationi tantum nec alio labore experta, nisi quod .... fraudibus et rapinis uicinarum quiete ${ }^{54}$ conturbans ${ }^{55}$.», Jordanès, Get., 123.

Pour la même confusion, voir Jordanès, Get., 122.

Cfr. un autre passage de Jordanès; «nec alius cladi finis fuit quam nox dirimeret postremusque fugientium rex ipse ... humero saucius in armis suis referretur.», Rom., 155.

Cfr. Tite-Live, 4, 26, 12.

Dans les formules de lois du VII e siècle et du VIII ${ }^{e}$ siècle on rencontre également nisi ut, à la place de quam ut, exempli gratia:

«... ut in aliter transagere non possum, nisi ut integrum statum meum in uestrum debiam implecare seruicium.» Formulae Merouingici et Karolini aeui, Legum Sectio V, Formulae - B 19 (manuscrit du VIII' siècle) ${ }^{56}$.

Le complément d'exception est souvent employé à l'époque tardive.

Le plus fréquent introductif semble être nisi, (par exemple: Commodien, Instr., 37, 13; Priscillien, Tract., 10, 140; St. Augustin, Serm., 200, 41, etc.; Claudien Mamert, Stat. anim., 1, 24 (p. 85, 1. 5-7); ibid., 1, 24 (p. 85, 1. 24-5); ibid., 2, 7 (p. 128, 1. 18-19), etc.; Faustus Reiensis, Serm., 13 (p. 275, 1. 14); ibid., 23 (p. 314, 1. 13-14); ibid., 23 (p. 317, 1. 24); St. Fulgence, Aetat., p. 129, 3; Jordanès, Rom., 167, etc., etc.

Praeter est employé en tant qu'adverbe limitatif. Par exemple:

«uti posthac pueri cum patribus in curiam ne introeant, praeter ille unus

Papirius.», Aulu-Gelle, 1, 23, $13^{57}$.

Dans les textes tardifs, praeter est souvent attesté en qualité de préposition (régissant l'Accusatif) - voir Jordanès, Rom., 159; ibid., 247; ibid., 387, etc.

Ajoutons que, si Horace et Ovide sont, vraisemblablement, les premiers à employer praeter avec l'infinitif présent, cet emploi se retrouve à l'époque tardive (voir Venantius Fortunatus, Carm. 4, 26, 3258). Pour nisi suivie de l'infinitif, voir Salvien, Eccl., 1, 38.

Extra indiquant l'exception (préposition avec l'Accusatif) est assez fréquente aux époques postclassique et tardive ${ }^{59}$. Exempli gratia:

«extra ipsum ... diem ... nunquam ieiunatur.», Peregrinatio Aetheriae, 27, 1. 
Quant à EXCEPTO, introductif de grande précision sémantique, il continue à être employé à l'époque tardive, en tant qu'adverbe et également comme préposition. Par exemple:

- «Sportulae ... eorum uenirent, excepto eorum qui trans mare erunt.», dans C. I. L., VI, no. 10234, 1. 17.

- «Nepos dictus a genere quodam scorpionum qui natos suos consumit, excepto eum qui ...», St. Isidore de Séville, Et., 10, $193^{60}$.

Le complément comparatif-exceptif est introduit par quam, voir Faustus Reiensis: «nihil est utilius quam hoc tantum.», Serm., 29 (p. 339, 1. 22).

Voir aussi Jordanès:

«.... non aliter se quam malo rei publicae potuit uindicare.», Get., 167.

Pour ce qui est du complément comparatif-exceptif introduit par quam, voir également Jordanès, Rom., 158; ibid., 159, etc., etc. Parfois quam apparaît au lieu de nisi:

«Quid est compati quam cum alio pati?», Tertullien, Prax., 29.

Dans les textes latino-romanes de la phase primitive des langues romanes, nisi apparait souvent pour introduire le complément d'exception. Voici un passage de «Glosas Silenses» (la seconde moitié du $\mathrm{X}^{\mathrm{e}}$ siècle), chap. De sacrificio:

"Sacrificium pro malis rebus nullo modo debemus offrir nisi tantum pro uonis.» ${ }^{61}$

Voici un passage du «Diplôme du Comte de Castille» (environ 1030):

«Proinde presot ille comite tota Spelia, et non eis laxabit nisi mas hereditatelias....», passage traduit par R. Menendez Pidal de la manière suivante:

«Por ello tomó el conde toda Espeja, y no le dejó sino sus pequenas heredades.» ${ }^{62}$

En ce qui concerne les textes romans, la ligature exceptive pour le complément est souvent «si non». Voici un passage d'un texte juridique du XIII' siècle, rédigé en portugais:

«... e por esto non deuedes consentir que razoen en uossa corte uogados que seyan sordos ou mudos, ... nen monge, nen hermitan, senon (s.-en.: aquelos) en pleytos de seus moesteyros e que aian lecença de seu abbade e de seu mayor ....», Flores de direito, p. $14^{63}$.

Dans la citation supra, senon est employé tout d'abord pour introduire un complément, ensuite, juxtaposé au pronom relatif, sert à introduire la subordonnée d'exception.

«Si non», sous la forme "se non», apparaît souvent en italien, en tant qu'introductif du complément d'exception, voir Boccaccio, Decam., Giorn. Sesta, $9^{64}$.

Excepto semble avoir également survécu dans les langues romanes, voir, en sarde, exceptu (en témoignent des passages de Constitutiones, Statuta et Ordinationes de Ecclesia de Ottana, VI ${ }^{65}$ ).

En ce qui concerne la subordonnée d'exception, celle-ci est introduite d'habitude dans le latin administratif, juridique et religieux des pays catholiques, à l'époque comprise entre les années 800 et 1200, par les locutions suivantes: nisi forte, nisi si, nisi quando, nisi tum quando, nisi cum, nisi sicut, nisi quantum, nisi ubi, nisi quod/quia, nisi 
$u t .{ }^{66}$ Si non, en tant qu'introductif des subordonnées d'exception, est assez rare dans ces textes, compte tenu du fait qu'on imitait généralement les modèles classiques. ${ }^{67}$

Pour ce qui est des langues romanes, les ligatures exceptives introduisant des subordonnées sont, partiellement, héritées du latin populaire.

Voici un fragment de loi rédigée en espagnol (le texte date de 1247):

«... non puede ni deue uender ni empennar .... las heredades que él ha, ella biua estando, si no fore con atorgamiento e con grandosa uoluntat de so muller ....», Los fueros de Aragón, A $78^{68}$.

Voir aussi le passage en portugais cité supra.

Quam et les remplaçants de quam dans le latin populaire de l'époque tardive ont également survécu dans les langues romanes.

Il est possible que nisi quod et nisi quia soient héritées en portugais.

Parfois on a créé des locutions conjonctives dans les langues romanes sur le modèle des locutions latines. Les locutions du type: «fuera que» (espagnol ancien), «fors que» (français ancien) imitent le modèle latin (praeter quod, extra quod). Une locution comme «hor-mis que» du français est créée en français d'après le modèle de «hors que» (variante de «fors que»).

Des locutions comme: «à moins que» (français), «a menos que» (espagnol), «a meno che» (italien), «mai pupin dacã«(roumain), ou «à part que» (français), «part que») (provençal), «lãsând deoparte» (roumain) correspondent à certains modèles logiques plus ou moins communs aux langues romanes et ne sont pas héritées du latin.

Voici quelques passages d'un texte rédigé en sarde, en 1475:

- «Item statuimus qui nessunu Preideru non potat narrer missa, a minus qui hapat narradu matutinu ....», Constitutiones, Statuta et Ordinationes De Ecclesia de Ottana, II 69.

- « ... ne minus (s.-en.: potet) andare daenanti de su preladu portando cussas cosas supra sa dicta pena, reservadu si veneret dae caminu, qui non esseret a tempus de lassare cussas ,...» Constitutiones, Statuta et Ordinationes De Ecclesia de Ottana, XXXIII ${ }^{70}$.

Dans le même texte en sarde, reservadu apparaît en tant qu'introductif du complément d'exception:

« ... paghet soddos quimbe, reservadu dogni legitima iscusa.»

Constitutiones, Statuta et Ordinationes De Ecclesia de Ottana, VII ${ }^{71}$.

Ce qui est important c'est que les introductifs des subordonnées d'exception des langues romanes contiennent une «négation», exactement comme en latin (qu'il s'agisse de «si non», «nisi», ou de «(foras de», «excepto», «exceptat», «levat» ${ }^{72}$, «tantum», «minus»).

Sans doute, une certaine confusion s'est-elle passée également dans les langues romanes entre les ligatures comparatives et celles exceptives proprement dites. C'est ainsi qu'on décèle souvent dans les textes romanes de toutes les époques l'emploi de "si non" à la place de que comparative, ou à la place de "que - non" ayant la même valeur (que comparative provenue de quam et de quod/quia comparatives du latin populaire tardif). 
Voici un passage de Foral da Guarda, texte juridique du XIIe siècle (en portugais):

«.... e nõ seruiã outro home senõ a seus senhores ....» 73 .

Voir, en roumain, le phénomène contraire: l'extension de l'emploi de la conjonction «decât» initialement à fonction comparative. «Decât» sert ensuite en roumain à introduire la subordonnée exceptive et le complément d'exception proprement dits.

Ajoutons que les langues romanes connaissent une évolution similaire par rapport au latin pour ce qui est des ligatures exceptives. On a choisi les mots et les locutions à haut degré de précision sémantique et on a réalisé, par étapes, l'unification des ligatures du complément et de la subordonnée correspondante.

Pour résumer, la subordonnée d'exception est fréquente en latin à toutes les époques, dans le registre populaire et dans le registre cultivé.

L'histoire des subordonnées d'exception a commencé, en grec ancien et en latin, par l'isolation d'une condition. Nisi, conjonction qui avait déjà acquiert un fort sens restrictif dans la période conditionnelle, a été considérée comme adéquate pour introduire la subordonnée d'exception. Bientôt nisi a été employée pour exprimer l'exclusion d'un fait. Les subordonnées comparatives introduites par quam, en dépendance des verbes négatifs, indiquent l'exclusion d'un fait.

$N i s i$ et quam qui pouvaient être facilement confondues avec le subordonnant de la conditionnelle et avec celui de la comparative et qui n'étaient point aptes à rendre l'exclusion de toute sorte de circonstances commencent bientôt à être accompagnée de différentes conjonctions: quod/quia; ut; cum, etc., en conformité avec le type de circonstance exceptée. Au niveau de la proposition, c'est le cas du substantif, ou du syntagme nominal, et assez souvent la préposition ou l'adverbe exceptif qui nous indiquent que l'exception porte sur un complément de manière, ou sur un complément de cause, et ainsi de suite.

A l'époque préclassique, les structures grammaticales et lexicales de la subordonnée d'exception prennent déjà forme. Doublement subordonnée, la proposition exceptive est d'habitude introduite par des locutions à sens précis, composées d'un adverbe restrictif et d'une conjonction de subordination. L'indicatif de la réalité est beaucoup employé. Dans la régente il y a, parfois, des corrélatifs.

Le nombre des locutions conjonctives augmente à l'époque classique et à l'époque postclassique, surtout dans le registre cultivé. On rencontre dans les textes aussi des pléonasmes, fréquents - selon toute vraisemblance - dans le latin populaire. Ces pléonasmes sont formés parfois par l'accumulation d'adverbes restrictifs, d'autres fois par l'agglomération de conjonctions.

Sans que l'incidence des propositions exceptives diminue dans le latin populaire de la basse époque, on y constate la réduction du nombre des locutions introductives. Dans le latin populaire et même dans le latin cultivé, on s'est orienté vers les mots et les locutions à haut degré de précision sémantique (tels que «si non» et «excepto»).

A part la réduction du nombre des locutions exceptives, on remarque qu'à l'époque tardive continuent à coexister les deux types de subordonnées: 
1. Les subordonnées d'exception proprement dite;

2. Les propositions comparatives-exceptives.

Les subordonnées exceptives proprement dites sont elles aussi, en dernière analyse, des subordonnées comparatives.

Il est aussi à noter l'existence d'un processus d'unification des éléments introductifs. Le cas de nisi est impressionnant: au début de l'histoire des subordonnées d'exception, nisi indiquait l'exclusion d'une condition; ensuite, cette conjonction a été employée pour l'isolation d'un fait. Devenu adverbe exceptif, nisi sert à introduire le complément d'exception. Chez Cicéron (par exemple dans Laelius), nisi est le plus fréquent introductif d'exception. A l'aide de nisi on a formé, à partir de l'époque préclassique, différentes locutions à sens précis.

Praeter, initialement adverbe et préposition régissant l'Accusatif, apparaît également employé dans le domaine de la subordination (seul, ou juxtaposé à quam).

Important est aussi l'adverbe excepto, employé d'abord pour introduire le complément d'exception, ensuite, dans des locutions, pour rendre l'isolation d'un fait, d'une condition, ou - rarement - d'une période de temps.

Le processus d'unification des ligatures exceptives a eu lui aussi pour résultat la disparition de certains adverbes et conjonctions (voir praeter quam, extra quam).

Les langues romanes héritent du latin populaire de l'époque tardive certains adverbes, conjonctions et locutions exceptifs (excepto et ses locutions; si non; quam et les remplaçants de quam dans le latin populaire de l'époque tardive).

Souvent on a copié sur le modèle latin et de nouveaux adverbes, locutions adverbiales et conjonctives sont apparus.

Nécessaires non seulement dans le langage juridique, administratif, ecclésiastique et scientifique, mais encore dans les oeuvres littéraires (comme par exemple Demanda do Santo Graall, manuscrit en portugais du XIV ${ }^{\mathrm{e}}$, ou du XV $\mathrm{X}^{\mathrm{e}}$ siècle $^{74}$ ) et dans le langage familier et populaire, le complément d'exception et la subordonnée correspondante sont continuellement attestés dans les textes romanes, parfois à partir même de leurs premiers manuscrits.

Seuls les adverbes, les conjonctions et les locutions à un haut degré de précision sémantique se sont conservés au cours des siècles (comme par exemple: excepto, si non, quam). La tendance à unifier les éléments introductifs du complément et de la subordonnée d'exception a également conduit au choix des mots et des locutions précis.

Le complément d'exception et la subordonnée d'exception sont, par leur essence, des circonstants comparatifs. A en juger d'après les vers suivants de Jean de La Fontaine:

«Un lièvre en son gîte songeait,

Car que faire en un gîte, à moins que l'on ne songe?»,

II, 14 - Le lièvre et les grenouilles, v. 1-2. 


\section{NOTES}

${ }^{1}$ Pour ce qui est des sens de si et de nisi, voir R. Iordache, Subordonata condițonală în latina clasică, dans «Lumea veche», Bucureşti, 1997, pp. 51-2, pp. 58-9; voir aussi R. Iordache, Remarques sur les raisons de la conservation de la conjonction latine 'si' dans les langues romanes, dans «Linguistica», 28, Ljub-ljana, 1988, pp. 36-40.

${ }^{2}$ Ch. E. Bennett, Syntax of early Latin, vol. I, Boston, 1910, p. 239.

3 Voir sur cette question le commentaire de G. Quaglia, l'édition de Laelius (Collezione diretta da R.Cantarella et B. Riposati), 1993, p. 48; voir aussi A. Draeger, Historische Syntax der lateinischen Sprache, vol. II -4, Leipzig, 1881, p. 650.

${ }^{4}$ Locution mentionnée dans J. B. Hofmann - A. Szantyr, Lateinische Grammatik, II -2, München, 1972, p. 5834, par. $314 b$.

5 Voir R. Kühner - C. Stegmann - A. Thierfelder, Ausführliche Grammatik der lateinischen Sprache, II -2, Hannover, 1971, p. $417^{1}$; P. Mc Glynn, Lexicon Terentianum, vol. I, London-Glasgow, 1963, p. 407.

${ }^{6}$ Pour ce qui est de extra quam si, voir Fr. Stolz - J. G. Schmalz, Lateinische Grammatik, München, 1928, p. 732.

${ }^{7}$ Exemple tiré de R. Kühner - C. Stegmann - A. Thierfelder, op. cit. II -1, p. 559.

${ }^{8}$ Exemples empruntés à A. Draeger, op. cit., II -4, p. 649.

${ }^{9}$ Exemples empruntés à A. Draeger, op. cit., II -4, p. 234.

10 Selon Oxford Latin Dictionary, fasc. 6, Oxford, 1977, p. 1447.

11 Voir H. Merguet, Lexicon zu den Schriften Caesars, Jena, 1886.

12 Voir Fr. Stolz - J. G. Schmalz, op. cit., p. 727.

13 Voir A. Draeger, op. cit., II -4, p. 233; voir R. Kühner - C. Stegmann - A. Thierfelder, op. cit., II -2, p. 416, 6; H. Merguet, Lexicon su den Schriften Caesars, op. cit.

14 Voir R. Kühner - C. Stegmann - A. Thierfelder, op. cit., II -2, p. $417^{2}$.

15 Pour d'autres exemples voir H. Merguet, Lexicon su den Reden des Cicero, vol. 3, Jena, 1882, p. 312.

16 Selon H. Merguet, Lexicon zu den Schriften Caesars, op. cit., p. 671.

17 Pour d'autres exemples voir R. Kühner - C. Stegmann - A. Thierfelder, op. cit., II -2, p. $417^{1}$.

18 Exemples empruntés à Fr. Stolz-J. G. Schmalz, op. cit., p. 732; voir également J. B. Hofmann - A. Szantyr, op. cit., II -2, p. 5952.

19 Selon J. B. Hofmann - A. Szantyr, op. cit., II -2, p. 2444.

20 Exemple tiré de Oxford Latin Dictionary, fasc. 6, op. cit., p. 1445.

21 Exemple tiré de R. Kühner - C. Stegmann - A. Thierfelder, op. cit., II -1, p. 5771.

22 Exemples empruntés à A. Draeger, op. cit., p. 234, par. 383; voir aussi J. B. Hofmann - A. Szantyr, op. cit., II -2, p. 5834.

23 Voir Thesaurus linguae Latinae, V -2, fasc. 13, Leipzig, 1953, p. 2059, 1. 44 sqq.; voir aussi Wilh. Freund N. Theil, Grand dictionnaire de la langue latine, vol. I, Paris, 1929, p. 1028.

24 Nisi, par extension de sens, est parfois employé au sens de solum, ou de modo (voir Varron, L. L., 5, 166).

25 Exemple emprunté à F. Gaffiot, Dictionnaire illustré Latin-Français, Paris, 1934.

${ }^{26}$ La ligature nisi uero si apparaît dans l'édition de H. Bornecque, Cicéron, vol. X, Paris, 1926; la même locution est présente dans l'édition de H. Kasten, Cicero's Staatsreden, Akademieverlag, vol. I, Berlin, 1972. Pour le même passage, A. Draeger propose la locution nisi uero (op. cit., II -4, p. 7521).

${ }^{27}$ Pour d'autres exemples de l'emploi de quam à la place de nisi et vice versa, voir J. B. Hofmann - A. Szantyr, op. cit., II -2 , pp. 595-6 1 .

28 Voir W. von Wartburg - P. Zumthor, Précis de syntaxe du français contemporain, Paris, 1973, p. 106. 
29 Quant à forsitan, voir H. Goelzer, Le latin de S. Avit, Paris, 1909, p. 358.

30 Voir R. Kühner - C. Stegmann - A. Thierfelder, op. cit., II -2, p. $416^{4}$, par. 217,5 e; voir également A. Draeger, op. cit., II -4 , pp. 752-3.

31 Pour ce qui est de l'importance du terme de référence, voir D. Irimia, Gramatica limbii române, Iassy, 1997, p. 464. Voir également D.Irimia pour la diversité des types de complément - termes de référence en roumain (ibid., pp. 464-5).

32 Voir, sur cette question, R. Iordache, Aclaraciones en torno al 'ut concesivo' $y$ al origen de la subordinada concesiva, dans «Helmantica», no. 110, Salamanque, 1985, p. 229; voir R. Iordache, Les subordonnées de manière en latin, Bref Plaidoyer pour la Syntaxe Historique, dans «Živa antika», vol. 48, Skopje, 1998, p. 70; R. Iordache, Subordonata conditională în latina clasică, dans «Lumea veche», vol. 3, București, 1997, pp. 51-2; R. Iordache, La parataxe conditionnelle: Indicatif, ou Impératif?, Bref Plaidoyer pour la Snytaxe Historique, dans «Živa antika», vol. 43, Skopje, 1993, pp. 47-55.

33 Voir F. Vanț-Stef, Sintaxa structurală a limbii vechi greceşti, Bucureşti, 1981, pp. 448-9.

34 Graphie erronée, au lieu de Carthago.

${ }^{35}$ L'exemple de Terentianus Maurus est emprunté à J. B. Hofmann-A. Szantyr, op. cit., II -2, p. $667^{1}$.

36 Voir R. Kühner - C. Stegmann - A. Thierfelder, op. cit., II -2, pp. 416-7.

37 Pour ce qui est des occurrences existantes chez Lactance, voir J. B. Hofmann - A. Szantyr, op. cit., Il -2, p. $244^{4}$, par. 132 (Zusätze); voir aussi R. Kühner - C. Stegmann - A. Thierfelder, op. cit., II -1, p. $576^{4}$.

38 Exemples empruntés à Oxford Latin Dictionary, fasc. 6, op.cit., p. 1447.

${ }^{39}$ Voir Thesaurus linguae Latinae, $\mathrm{V}-2$, fasc. 13, op. cit., p.2054, 1.21 sqq.

40 Pour d'autres données, voir J. B. Hofmann - A. Szantyr, op.cit., II -2, p. $583^{4}$.

41 D'après A. Draeger, op.cit., II -4, p. 649.

42 Pour ce qui est de la fonction sélective et qualificative des adverbes ut, utpote, quippe, quidem, praesertim, juxtaposés au pronom relatif, voir R. Iordache, Relatives causales, ou Relatives consécutives?, Bref Plaidoyer pour la Syntaxe Historique, dans «Helmantica», no. 86, Salamanque, 1977, pp. 269-73.

43 Voir Thesaurus linguae Latinae, V -2, Leipzig, 1910, p. 1249, 1. 29-31.

44 Voir Thesaurus linguae Latinae, $V$-2, op. cit. - note antérieure, p. 1249, 1. 32-34.

45 VoirThesaurus linguae Latinae, V -2, op. cit., p. 1249, 1. 34 sqq.

46 Voir Thesaurus linguae Latinae, V -2, op. cit., p. 1249, 1. 37 sqq.

${ }^{47}$ Exemple emprunté à Fr. Stolz - J. G. Schmalz, op. cit., p. 727.

48 Pour ce qui est de l'emploi de nisi quia et de nisi quod dans Itala et Vulgata, voir Fr. Stolz - J. G. Schmalz, op. cit., p. 727.

49 Voir J. B. Hofmann - A. Szantyr, op. cit., II -2, p. $583^{2}$.

50 Occurrences présentées en ordre chornologique.

${ }^{51}$ Voir Fr. Stolz - J. G. Schmalz, op. cit., p. 727; J. B. Hofmann - A. Szantyr, op. cit., II -2, p. 5874 . Voir pourtant l'opinion de J. Pedro Machado, Dicionário etimolügico da língua portuguesa, vol. III, Lisboa, 1967, p. 1654: «etimologia controversa».

52 Voir J. B. Hofmann - A. Szantyr, op. cit., II -2, p. $244^{4}$.

53 Voir note 22.

54 quiete - Accusatif singulier à l'omission de $-m$ final.

55 Participe présent au lieu de l'indicatif imparfait - phénomène assez fréquent chez Jordanès et chez d'autres auteurs tardifs. Voir, sur cette question, Fr. Werner, Über die Latinität der 'Getica' des Iordanes, Halle, 1908, p. 89; voir pour Victor Vitensis, Index uerborum et locutionum, dans C. S. E. L., vol. 7, Vienne, 1881, p. 165. 
56 Texte renfermant beaucoup d'erreurs par rapport au latin cultivé: in aliter, à la place de aliter; transagere, au lieu de transigere; confusion entre - $i$ - et -e-(voir debiam et implecare); seruicium, à la place de seruitium. Texte reproduit selon l'édition de K. Zeumer, Hannover, 1886, dans «M. G. h.».

57 Exemple tiré de Oxford Latin Dictionary, fasc. 6, op. cit., p. 1445.

58 Voir J. B. Hofmann - A. Szantyr, op. cit., II -2, p. $245^{1}$.

59 Voir Thesaurus linguae Latinae, V -2, fasc. 13, op. cit., p. 2059, 1. 57 sqq.

60 Pour d'autres références, voir Thesaurus linguae Latinae, V-2, op. cit., p. 1249, 1. 39 sqq. Le passage de St. Isidore est reproduit selon l'édition des Professeurs J. Oroz Reta et M. A. Marcos Casquero, St. Isidoro de Sevilla, Etimologias, Madrid, 1993, vol. I.

61 D'après R. Menendez Pidal, El idioma espanol en sus primeros tiempos, Madrid, 1964, pp. 22-23.

62 Voir R. Menendez Pidal, op. cit., pp. 20-1.

63 Selon l'édition de J. J. Nunes, Crestomatia arcaica, Lisboa, 1959.

Pour ce qui est de senõ introduisant le complément d'exception en portugais ancien, voir également Foral da Guarda, dans J. J. Nunes, Crestomatia arcaica, op. cit., pp. 3-4.

64 D'après l'édition de A. Fr. Massera, vol. 1, Bari, 1927.

65 Selon l'édition de G. Spano, Ortografia sarda nazionale, vol. II, Cagliari, 1840, p. 99.

66 Voir Fr. Blatt, Nouum Glossarium Mediae Latinitatis, Copenhague, 1967, p. 1271, 1. 3-54; ibid., p. 1272, 1. 1-14.

67 Pour des occurrences de «si non» à cette époque, voir Fr. Blatt, op. cit., p. 1359, 1. 36 sqq.

68 Selon l'édition de G. Tilander, Los fueros de Aragón, según el manuscrito 458 de la Biblioteca Nacional de Madrid, Lund, 1937.

${ }^{69}$ D'après l'édition de G. Spano, op. cit., Vol. II, p. 99.

${ }^{70}$ Selon l'édition de G. Spano, op. cit., Vol. II, p. 101.

71 Selon l'édition de G. Spano, op. cit., Vol. II, p. 99.

72 levat que - locution exceptive en provençal.

73 Selon l'édition de J. J. Nunes, Crestomatia arcaica, op. cit.

74 Voir l'édition de J. Leite De Vasconcellos, Textos arcaicos, Lisboa, 1922, p. 44.

\section{Povzetek \\ OPOMBE O IZVZEMALNIH PODREDNIH STAVKIH V LATINŠČINI IN ROMANSKIH JEZIKIH}

Izvzemalni stavki, torej stavki, ki izražajo izvzemanje ali izjemo, se v večini latinskih slovnic obravnavajo deloma kot pogojni, deloma kot primerjalni odvisniki. Avtorica jih združuje v eno skupino $\mathrm{z}$ dvema podskupinama: odvisniki $\mathrm{z}$ veznikom nisi in odvisniki $\mathrm{z}$ veznikom quam. Novo skupino utemeljuje predvsem $z$ oblikovnimi skupnimi značilnostmi in se ne naslanja samo na skupne pomenske poteze. Članek ob številnih primerih spremlja razvoj odvisnikov od stare latinščine preko klasične dobe $\mathrm{v}$ pozno antiko, srednji vek in romanske jezike. V drugem delu se obravnava razvoj primerjalnih odvisnikov na podlagi hipoteze, da so pogojni odvisniki po izvoru primerjalni. V razvojnem prikazu izvzemalnih odvisnikov $\mathrm{z}$ veznikom nisi avtorica izhaja iz pomenske prestavitve $\mathrm{v} v \mathrm{ve}$ bini pogojnega odvisnika: odvisnik z nisi je prvotno izražal zanikan pogoj, nato zanikano dejstvo. 